\title{
artigo
}

Trindade, R.C.G.; Rodrigues, M.R.K.; Molina, A.C.; Lima, S.A.M.

Conhecimento dos profissionais de enfermagem sobre as atividades desenvolvidas pelo gerenciamento de risco em hospital do interior paulista

\section{Conhecimento dos profissionais de enfermagem sobre as atividades desenvolvidas pelo gerenciamento de risco em hospital do interior paulista}

\author{
Knowledge of nursing professionals on the activities developed by risk management in a hospital in the interior of \\ São Paulo
}

Conocimiento de profesionales de enfermería sobre las actividades desarrolladas por la gestión de riesgos en un hospital del interior de São Paulo

\begin{abstract}
RESUMO
Objetivo: avaliar o conhecimento dos profissionais de enfermagem sobre as atividades desenvolvidas pelo Gerenciamento de Risco (GR) em hospital escola do interior paulista. Método: estudo exploratório realizado em 2013 com análise descritiva quantitativa dos dados. Foi aplicado um questionário aos profissionais de enfermagem, que trabalhavam nas unidades do hospital. Resultados: a maioria dos profissionais de enfermagem conhecia a unidade de GR $(90,60 \%$ dos enfermeiros e $82,30 \%$ dos Técnicos e Auxiliares de Enfermagem);71,90\% dos Enfermeiros e 35,45\% dos Técnicos e Auxiliares de Enfermagem referiram ter realizado notificação na instituição. Os profissionais entrevistados relataram ter presenciado ocorrência de eventos adversos que não foram notificados ao GR (Enfermeiros=37,50\% e Técnicos e Auxiliares de Enfermagem=29,10\%). A maioria dos profissionais de enfermagem indicou a necessidade de treinamento (Enfermeiros=78,10\% e Técnicos e Auxiliares de Enfermagem=88,60\%). Conclusão: necessário adotar estratégias para melhorar o conhecimento dos profissionais e o processo de notificação de produtos de saúde na instituição.
\end{abstract}

DESCRITORES: Gestão de Riscos; Vigilância de Evento Sentinela; Equipe de Enfermagem.

\section{ABSTRACT}

Objective: to evaluate the knowledge of nursing professionals about the activities developed by Risk Management (GR) at the São Paulo State Hospital. Method: exploratory study conducted in 2013 with quantitative descriptive analysis of the data. A questionnaire was applied to nursing professionals who worked in the hospital units. Results: most nursing professionals knew the GR unit $(90.60 \%$ of nurses and $82.30 \%$ of nursing technicians and assistants); $71.90 \%$ of nurses and $35.45 \%$ of nursing technicians and assistants reported having notified the institution. The professionals interviewed reported having adverse events that were not notified by the GR (Nurses $=37.50 \%$ and Nursing Technicians and Assistants $=29.10 \%$ ). Most nursing professionals indicate a need for training (Nurses $=\mathbf{7 8 . 1 0 \%}$ and Nursing Technicians and Assistants $=88.60 \%$ ). Conclusion: It is necessary to adopt strategies to improve professionals' knowledge and the process of notification of health products in the institution.

DESCRIPTORS: Risk Management; Sentinel Surveillance; Nursing, Team.

\section{RESUMEN}

Objetivo: evaluar el conocimiento de los profesionales de enfermería sobre las actividades desarrolladas por Risk Management (GR) en el Hospital del Estado de São Paulo. Método: estudio exploratorio realizado en 2013 con análisis descriptivo cuantitativo de los datos. Se aplicó un cuestionario a los profesionales de enfermería que trabajaban en las unidades hospitalarias. Resultados: la mayoría de los profesionales de enfermería conocían la unidad GR (90,60\% de las enfermeras y $82,30 \%$ de los técnicos y asistentes de enfermería); El $71.90 \%$ de las enfermeras y el $35.45 \%$ de los técnicos y asistentes de enfermería informaron haber notificado a la institución. Los profesionales entrevistados informaron haber tenido eventos adversos que no fueron notificados por el GR (enfermeras $=37.50 \%$ y técnicos y asistentes de enfermería $=29.10 \%$ ). La mayoría de los profesionales de enfermería indican una necesidad de capacitación (enfermeras $=78.10 \%$ y técnicos y asistentes de enfermería $=88.60 \%$. Conclusión: es necesario adoptar estrategias para mejorar el conocimiento de los profesionales y el proceso de notificación de productos de salud en la institución.

DESCRIPTORES: Gestión de Riesgos; Vigilancia de Guardia; Grupo de Enfermería.

RECEBIDO EM: 15/12/2019 APROVADO EM: 15/12/2019

\section{Romayne Caroline Giandoni Trindade}

Enfermeira. Aprimoramento Profissional em Hematologia e Hemoterapia pela Faculdade de Medicina de Botucatu, UNESP-Universidade Estadual Paulista - Botucatu, SP, Brasil. https://orcid.org/0000-0002-9096-8917 


\section{Meline Rossetto Kron Rodrigues}

Enfermeira.Professora Doutora do Programa de Pós-graduação Stricto Sensu em Enfermagem da Universitas - UNG, GuaruIhos, SP, Brasil. https://orcid.org/0000-0003-2174-268X

\section{Ana Claudia Molina}

Enfermeira. Prefeitura Municipal de Botucatu. Mestre e Doutora pela Faculdade de Medicina de Botucatu, UNESP-Universidade Estadual Paulista - Botucatu, SP, Brasil. https://orcid.org/0000-0002-2934-7484

\section{Silvana Andréa Molina Lima}

Enfermeira. Professora Associada do Departamento de Enfermagem da Faculdade de Medicina de Botucatu, UNESP-Universidade Estadual Paulista - Botucatu, SP, Brasil. https://orcid.org/0000-0001-9945-2928

\section{Introdução}

A falta de qualidade dos produtos hospitalares e assistência ao paciente, bem como erros ou falhas, podem gerar agravos à saúde e até possíveis sequelas e/ou morte ${ }^{(1)}$.

Neste contexto, nota-se a busca constante pela qualidade desses produtos hospitalares e da assistência prestada ao paciente para garantir segurança aos pacientes e profissionais de saúde.

Em 1999, o Ministério da Saúde criou a Agência Nacional de Vigilância Sanitária (ANVISA), a qual "tem a responsabilidade e a missão de promover e proteger a saúde, garantindo segurança sanitária de serviços e produtos de saúde"(2).

Em 2001, a ANVISA criou o Projeto Hospitais Sentinela (PHS), que teve como objetivo a construção de uma rede de hospitais preparados para notificar eventos adversos e queixas técnicas de produtos de saúde, como: insumos, materiais, medicamentos, saneantes e equipamentos médico-hospitalares em uso no Brasil ${ }^{(2,3)}$.

A partir desse projeto, houve a implantação da Unidade de Gerenciamento de Risco Sanitário nas instituições de saúde selecionadas, podendo assim responder a necessidade da ANVISA, em obter informação qualificada de vigilância pós-comercialização envolvendo produtos de saúde ${ }^{(2,3)}$.

O PHS deu início ao monitoramento da ocorrência de eventos adversos e queixas técnicas em quatro grandes áreas: Farmacovigilância, Tecnovigilância, Hemovigilância e Saneantes.

A Farmacovigilância visa atender me- tas e ações para a ampliação de diretrizes que garantam a detecção precoce e controle de riscos, a fim de prevenir eventos adversos e a ocorrência de desvio de qualidade e ineficácia terapêutica, entre outras ocorrências indesejáveis relacionadas aos medicamentos ${ }^{(4)}$.

A Tecnovigilância desenvolve ações de vigilância que envolvem materiais e artigos descartáveis, por meio de notificações de suspeitas de queixas técnicas e eventos adversos ${ }^{(5)}$.

A Hemovigilância tem a finalidade de investigar, analisar, prevenir e processar informações de possíveis reações imediatas ou tardias e efeitos colaterais causados por componentes sanguíneos denominados de reações transfusionais ${ }^{(6)}$.

A área de Saneantes visa detectar, avaliar e prevenir os efeitos adversos ou quaisquer problemas relacionados aos saneantes de uso hospitalar ${ }^{(2)}$.

Mediante o exposto, verifica-se a necessidade de identificar o conhecimento dos profissionais de enfermagem sobre o Gerenciamento de Risco nas instituições de saúde e as atividades de vigilância pós-comercialização desenvolvidas, justificando a execução desse estudo.

Objetivou-se avaliar o conhecimento dos profissionais de enfermagem sobre as atividades desenvolvidas no Gerenciamento de Risco em hospital do interior paulista.

\section{METODOLOGIA}

Trata-se de um estudo do tipo exploratório, descritivo com análise quantitativa dos dados realizado no ano de 2013.

Este estudo foi realizado nas unidades de um hospital escola do interior do Estado de São Paulo, incluindo profissionais da área da saúde (enfermeiros, técnicos e auxiliares de enfermagem).

Os critérios de inclusão para o estudo foram: ser enfermeiro e técnico ou auxiliar de enfermagem do Hospital estudado e concordar em participar da pesquisa.

A amostra foi constituída de 111 profissionais de enfermagem, sendo 32 enfermeiros e 79 auxiliares e técnicos de enfermagem. A coleta de dados foi realizada por meio de preenchimento de formulário pelos participantes, sem identificação dos mesmos. O formulário constou de perguntas relacionadas ao cargo, tempo na instituição, local de trabalho (dividido em unidade de longa permanência para local de internação e unidade de curta permanência para local que os pacientes não permanecem internados), necessidade de maior conhecimento sobre o assunto e da unidade de Gerenciamento de Risco, realização de notificação, se presenciou ocorrência que não foi notificada e temas para treinamento na área.

Os dados coletados foram tabulados em planilha Microsoft Excel. Foi realizada a análise descritiva dos dados, a partir de tabelas de distribuição de frequências absoluta e relativa. Foi utilizado o modelo logístico ajustado para explicar a chance de ter realizado uma notificação, de conhecer a Unidade de Gerenciamento de Risco e de ter presenciado alguma ocorrência que não foi notificada.

Foram preservados os aspectos éticos previstos na Resolução n. ${ }^{\circ}$ 466/12 do Conselho Nacional de Saúde (CNS). A pesquisa foi aprovada pelo Comitê de Ética em Pesquisa da instituição estudada 


\section{artigo}

Trindade, R.C.G.; Rodrigues, M.R.K.; Molina, A.C.; Lima, S.A.M.

Conhecimento dos profissionais de enfermagem sobre as atividades desenvolvidas pelo gerenciamento de risco em hospital do interior paulista

sob parecer n. ${ }^{\circ} 4114-2012$.

\section{RESULTADOS}

No período em estudo foram entrevistados 111 profissionais de enfermagem, sendo 32 Enfermeiros e 79 Técnicos e Auxiliares de Enfermagem, e suas características foram identificadas e apresentadas na Tabela 1.

$\mathrm{Na}$ distribuição dos profissionais entrevistados, de acordo com o tempo

Tabela 1. Perfil da amostra dos profissionais entrevistados. Botucatu, SP, Brasil, $2013(n=111)$

\begin{tabular}{|c|c|c|c|c|}
\hline \multirow[b]{2}{*}{ VARIÁVEL } & \multicolumn{2}{|c|}{$\begin{array}{l}\text { ENFERMEIRO } \\
\quad \mathrm{N}=32\end{array}$} & \multicolumn{2}{|c|}{$\begin{array}{c}\text { AUXILIAR/TÉCNICO } \\
\text { N=79 }\end{array}$} \\
\hline & $\mathbf{N}$ & $\%$ & $\mathbf{N}$ & $\%$ \\
\hline \multicolumn{5}{|l|}{ Tempo na instituição } \\
\hline 1 a 5 anos & 16 & 50,00 & 16 & 20,25 \\
\hline 6 a 10 anos & 03 & 9,40 & 10 & 12,66 \\
\hline 11 a 15 anos & 04 & 12,50 & 07 & 8,87 \\
\hline 16 a 20 anos & 05 & 15,60 & 12 & 15,18 \\
\hline 21 a 30 anos & 04 & 12,50 & 34 & 43,04 \\
\hline \multicolumn{5}{|l|}{ Local de trabalho } \\
\hline Unidade de Longa Permanência & 18 & 56,25 & 40 & 50,60 \\
\hline Unidade de Curta Permanência & 14 & 43,75 & 39 & 49,40 \\
\hline \multicolumn{5}{|l|}{ Conhece o HS } \\
\hline Não & 03 & 9,40 & 14 & 17,70 \\
\hline Sim & 29 & 90,60 & 65 & 82,30 \\
\hline \multicolumn{5}{|l|}{$\begin{array}{l}\text { Necessidade de mais conhecimento } \\
\text { sobre as atividades do HS }\end{array}$} \\
\hline Não & 07 & 21,90 & 17 & 21,50 \\
\hline $\operatorname{Sim}$ & 25 & 78,10 & 62 & 78,50 \\
\hline \multicolumn{5}{|l|}{ Já realizou notificação } \\
\hline Não & 09 & 28,10 & 51 & 64,55 \\
\hline $\operatorname{Sim}$ & 23 & 71,90 & 28 & 35,45 \\
\hline \multicolumn{5}{|l|}{ Já presenciou ocorrência não notificada } \\
\hline Não & 20 & 62,50 & 56 & 70,90 \\
\hline Sim & 12 & 37,50 & 23 & 29,10 \\
\hline \multicolumn{5}{|l|}{ Motivo da ocorrência não notificada } \\
\hline Desconhecimento do processo de notificação & 06 & 33,30 & 13 & 46,43 \\
\hline Insegurança/Medo & 02 & 11,10 & 00 & 0,00 \\
\hline Falta de Tempo & 03 & 16,70 & 02 & 7,14 \\
\hline Apenas chefia pode notificar & 04 & 22,20 & 06 & 21,43 \\
\hline Ausência de formulário & 00 & 0,00 & 03 & 10,70 \\
\hline Ter ocorrido com outro profissional & 03 & 16,70 & 03 & 10,70 \\
\hline Ter notificado e não ter resolvido o problema & 00 & 0,00 & 01 & 3,60 \\
\hline Total: & 18 & 100 & 28 & 100 \\
\hline
\end{tabular}

de trabalho na instituição, verificou-se que metade dos Enfermeiros possuía de 1 a 5 anos de trabalho na instituição e 43,04\% dos Técnicos e Auxiliares de Enfermagem possuíam de 21 a 30 anos de trabalho.

Os profissionais entrevistados trabalhavam em unidades de longa permanência, sendo 56,25\% dos Enfermeiros e 50,60\% dos Técnicos e Auxiliares de Enfermagem; e em Unidades de curta permanência, sendo $43,75 \%$ dos Enfermeiros e $49,40 \%$ dos Técnicos e Auxiliares de Enfermagem.

Em relação ao conhecimento das atividades Unidade de Gerenciamento de Risco na instituição estudada, a maioria relatou conhecer o GR (90,60\% dos enfermeiros e 82,30\% dos Técnicos e Auxiliares de Enfermagem). Entretanto, a maioria dos profissionais de Enfermagem, em ambas as categorias, referiu a necessidade de obter mais informações sobre as atividades do GR.

De acordo com a realização de notificação de queixas técnicas e eventos adversos, 71,90\% dos Enfermeiros e 35,45\% dos Técnicos e Auxiliares de Enfermagem referiram ter realizado notificação na instituição.

Os profissionais entrevistados relataram ter presenciado ocorrência de eventos adversos que não foram notificados ao GR (Enfermeiros $=37,50 \%$ e Técnicos e Auxiliares de Enfermagem =29,10\%).

Para os Enfermeiros, a ocorrência de eventos adversos não notificados foi relacionada ao desconhecimento do processo de notificação (33,30\%), atribuição do processo de notificação à chefia $(22,20 \%)$, falta de tempo (16,70\%), ocorrência com outro profissional $(16,70 \%)$ e insegurança ou medo (11,70\%). Já para os Técnicos e Auxiliares de Enfermagem, a subnotificação foi relacionada ao desconhecimento do processo de notificação (46,43\%), atribuição do processo de notificação à chefia $(21,43 \%)$, ocorrência com outro profissional (10,70\%), ausência e formulário (10,70\%), falta de tempo $(7,14 \%) \mathrm{e}$ ter notificado anteriormente, sem resolução do problema $(3,60 \%)$. 


\section{DISCUSSÃO}

A pesquisa realizada permitiu avaliar o conhecimento dos profissionais de enfermagem sobre as atividades desenvolvidas na Unidade de Gerenciamento de Risco em um hospital do interior paulista bem como do processo de notificação de produtos de saúde, contribuindo para identificação dos temas para orientação de treinamento a ser realizado no futuro.

Observou-se que metade da categoria dos Enfermeiros entrevistados possuía tempo de trabalho na instituição de 1 a 5 anos; já quase metade dos profissionais de nível técnico possuía tempo de trabalho na instituição acima de 20 anos. O tempo de trabalho entre as categorias poderia representar uma diferença de conhecimento do hospital como um todo e de experiência entre as categorias. Entretanto, a maioria dos Enfermeiros e Técnicos/Auxiliares de Enfermagem relatou ter conhecimento da existência do GR na instituição, embora necessitasse de maiores informações a respeito das atividades desenvolvidas. Dados semelhantes foram observados por outros autores ${ }^{(7,8)}$.

De acordo com modelo logístico ajustado, a chance do profissional Enfermeiro em conhecer o GR foi de 2,58 vezes maior que o Técnico ou Auxiliar de Enfermagem. Isto evidencia que o cargo de chefia ou papel de liderança do profissional Enfermeiro faz com ele tenha maior conhecimento sobre a importância e o processo de notificação dentro da instituição.

Estudos demonstram que o enfermeiro é o profissional que mais realiza notificações de ocorrências com produtos de saúde ${ }^{(9,10)}$. De acordo com Capucho $^{(11)}$, além dos enfermeiros estarem em maior número na instituição, eles permanecem maior tempo ao lado do paciente e possuem capacitação para realização dos registros. Por outro lado, estudo realizado com profissionais de enfermagem identificou que $76,8 \%$ nunca realizou uma notificação ${ }^{(8)}$.

\section{Estudos}

demonstram que

o enfermeiro é

o profissional

que mais realiza

notificações

de ocorrências

com produtos

de saúde ${ }^{(9,10)}$.

De acordo com

Capucho $^{(11)}$, além

dos enfermeiros

estarem em

maior número na

instituição, eles

permanecem maior

tempo ao lado do

paciente e possuem

capacitação para

realização dos

registros.
Em nosso estudo, dentre a equipe de enfermagem, a maioria dos Enfermeiros relatou realizar notificações de ocorrências ao GR quando comparado ao grupo de Técnicos/Auxiliares de Enfermagem. De acordo com o modelo logístico ajustado, a chance de o Enfermeiro ter realizado uma notificação foi de 9,26 vezes maior que o Técnico ou Auxiliar de Enfermagem. Isto pode ser explicado pelo fato de o profissional Enfermeiro assumir papel de líder da equipe, o que faz com que ele tenha maior conhecimento sobre as ocorrências e permita maior participação no processo de notificação dentro da instituição e centralização do papel de notificador.

Nesse estudo também foram identificados problemas ou dificuldades com a notificação de ocorrências de eventos adversos, isto é, uma parte dos profissionais presenciaram eventos não notificados, o que demonstra a ocorrência de subnotificação entre os profissionais de saúde da instituição estudada. Em trabalho realizado por Lima e colaboradores ${ }^{(12)}$, no mesmo hospital, também foi observada a subnotificação de eventos ocorridos com medicamentos.

A ocorrência de subnotificação foi atribuída pelos profissionais de enfermagem a diversos fatores, como: desconhecimento do processo de notificação, atribuição do processo de notificação à chefia, falta de tempo e ocorrência com outro profissional. Os Enfermeiros também relataram ocorrência de não notificação por insegurança ou medo; e os profissionais de nível técnico, por ausência e formulário e notificação anterior, sem resolução do problema.

Para Silva e colaboradores ${ }^{(13)}$, essa subnotificação pode ocorrer pelo fato dos profissionais dos hospitais identificarem apenas os Eventos Adversos Graves, não notificando os de menor gravidade quer seja por falta de mecanismos de identificação e registros ou medo de punição/exposição do profissional ${ }^{(13,14)}$ ou por falta de conhecimento ${ }^{(15)}$.

Por outro lado, eventos adversos não 


\section{artigo}

Trindade, R.C.G.; Rodrigues, M.R.K.; Molina, A.C.; Lima, S.A.M.

Conhecimento dos profissionais de enfermagem sobre as atividades desenvolvidas pelo gerenciamento de risco em hospital do interior paulista

são fáceis de serem identificados, quer seja por fatores relacionados ao paciente ou pela própria droga ou produto de saúde ${ }^{(12)}$. Outros fatores que interferem no processo de notificação são os aspectos culturais e organizacionais, a estrutura prática da assistência, os sistemas de segurança, os regulamentos e os processos de trabalho ${ }^{(16)}$.

Roque e colaboradores ${ }^{(17)}$ citam que os profissionais de saúde tendem a valorizar os eventos adversos que comprometem a vida do paciente, fazendo com que os eventos menos graves não sejam registrados no prontuário do paciente.

Neste sentido, a adoção de estratégias para redução da subnotificação se torna importante dentro dos serviços de saúde, contribuindo para a melho- ria da qualidade dos produtos e da assistência e garantindo maior segurança aos pacientes.

Poucos estudos semelhantes foram encontrados na literatura, o que não permitiu maiores comparações com os achados dessa pesquisa.

\section{CONCLUSÃO}

A maioria dos Enfermeiros e Técnicos/Auxiliares de Enfermagem relatou ter conhecimento da existência do GR na instituição. Entretanto, as duas categorias necessitam de maiores informações a respeito das atividades desenvolvidas.

Dentre a equipe de enfermagem, o Enfermeiro é o profissional que mais realiza notificações de ocorrências ao Hospital Sentinela.

Foi relatada a ocorrência de subnotificação pelos profissionais de enfermagem, sendo atribuída a diversos fatores, como: desconhecimento do processo de notificação, atribuição do processo de notificação à chefia, falta de tempo, ocorrência com outro profissional, insegurança ou medo, por ausência de formulário e notificação anterior, sem resolução do problema.

O estudo permitiu identificar a necessidade de adoção de estratégias para melhorar o processo de notificação de produtos de saúde pela unidade de GR e, com isso, garantir a qualidade e segurança aos profissionais de saúde e pacientes. -

\section{REFERÊNCIAS}

1.Mendes W, Travassos C, Martins M, Noronha JC. Revisão dos estudos de avaliação da ocorrência de eventos adversos em hospitais. Rev Bras Epidemiol 2005; 8(4): 393-406.

2. Ministério da Saúde, Agência Nacional de Vigilância Sanitária (BR). Áreas de atuação: Tecnovigilância, Farmacovigilância, Saneantes e Hemovigilância [Internet]. 2008 [acesso em 02 set 2008]. Disponivel em: http//:www.anvisa.org.br.

3. Spadella CT. Gerenciamento do risco sanitário hospitalar na área de tecnovigilância do HC da Unesp de Botucatu. Boletim Informativo - Projeto Hospitais Sentinela 2005; 20: 4-5.

4.Associação Nacional de Vigilância Sanitária [Internet]. 2010 [acesso em 29 jan 2010]. Disponível em: http://portal.anvisa.gov. br/wps/portal/anvisa/posuso/tecnovigilancia.

5.Projetti ABFC, Cioffi JGM. Hemovigilância: verificação final da qualidade da transfusão? Rev. bras. hematol. hemoter. 2008; 30 (3): 173-176.

6.Fakih FT, Freitas GF, Secoli SR. Medicação: aspectos ético-legais no âmbito da enfermagem. Rev. bras. Enferm 2009; 62(1): 132-135.

7.Silva-Batalha, EMS. A cultura de segurança do paciente na percepção de profissionais de enfermagem de um hospital de ensino. [dissertação]. São Paulo: Escola de Enfermagem, Universidade de São Paulo; 2012.

8. Massoco ECP. Percepção da equipe de enfermagem de um hospital de ensino acerca da segurança do paciente [dissertação]. São Paulo: Escola de Enfermagem da Universidade de São Paulo; 2012.

9.Capucho HC, Arnas ER, Cassiani SHB. Segurança do Paciente: comparação entre notificações voluntárias manuscritas e informatizadas sobre incidentes em Saúde. Revista Gaúcha de Enfer- magem. 2013; 34(1):164-72.

10.Bezerra ALQ, Silva AEBC, Branquinho NCSS, Paranaguá TTB. Análise de Queixas Técnicas e Eventos Adversos Notificados em um Hospital Sentinela. Revista de Enfermagem UERJ. 2009; 17(4):467-72.

11.Capucho HC. Sistemas manuscrito e informatizado de notificação voluntária de incidentes em saúde como base para a cultura de segurança do paciente [Tese de Doutorado]. Ribeirão Preto (SP): Universidade de São Paulo; 2012.

12. Lima PF, et al. Queixas técnicas e eventos adversos a medicamentos notificados em um hospital sentinela do interior de São Paulo, 2009-2010. Epidemiol. Serv. Saúde. 2013; 22 (4): 679686.

13. Silva AEBC, Reis AMM, Miasso Al, Santos JO, Cassiani SHB. Eventos adversos a medicamentos em um hospital sentinela do Estado de Goiás. Rev. Latino-Am. Enfermagem. mar-abr 2011;19(2):[09 telas].

14. Paiva MCMS, Popim RC, Melleiro MM, Tronchim DMR, Lima SCAM, Juliani CMCM. Motivos da equipe de enfermagem para a notificação de eventos adversos. Rev. Latino-Am. Enfermagem. 2014 set.-out.; 22(5):747-54.

15.Bezerra ALQ, Silva AEBC, Branquinho NCSS, Paranaguá TTB. Análise de Queixas Técnicas e Eventos Adversos Notificados em um Hospital Sentinela. Revista de Enfermagem UERJ. 2009; 17(4):467-72.

16. Moumtzoglou A. Factors impeding nurses from reporting adverse events. J Nurs Manage. 2010;18(5):542-7.

17. Roque KE, Melo ECP. Avaliação dos eventos adversos a medicamentos no contexto hospitalar. Esc Anna Nery. 2012; 16(1):121-127. 